\title{
Direct Evidence of a Role for Mast Cells in the Pathogenesis of Antigen-induced Bronchoconstriction
}

\author{
Thomas B. Casale, Douglas Wood, Hal B. Richerson, Brian Zehr, Donald Zavala, and Gary W. Hunninghake \\ Department of Internal Medicine, University of Iowa Hospitals and Clinics, Iowa City, Iowa 52242
}

\begin{abstract}
We measured bronchoalveolar lavage (BAL) fluid histamine levels in allergic asthmatics and nonallergic normal subjects after local airway antigen and cold $22^{\circ} \mathrm{C}$ normal saline challenges. Immediately after instillation of antigen through a bronchoscope wedged into a subsegmental airway, all 17 allergic asthmatics but none of the nine normal subjects had visible airway constriction. The asthmatics had a concomitant mean increase in BAL histamine of $23 \%(P=0.005)$, whereas the normals had no change in BAL histamine. Among the allergic asthmatics, the change in BAL histamine content in response to antigen directly correlated with the control (baseline) BAL histamine content $(r=0.66, P=0.003)$. Moreover, asthmatics with large antigen-induced changes in BAL histamine had greater airway methacholine sensitivity than did asthmatics without measurable increases in BAL histamine (8 \pm 2 vs. $41 \pm 31$ breath units). Neither asthmatics nor normal subjects had airway constriction or changes in BAL histamine levels in response to nonspecific challenge with cold saline. Our data suggest that when allergic asthmatics are exposed to relevant antigens they have in vivo lung mast cell degranulation which results in airway constriction and contributes to nonspecific airway hyperresponsiveness.
\end{abstract}

\section{Introduction}

Inhalation of antigen to which an allergic asthmatic is sensitized often results in both early and late asthmatic responses which reach maximum 15-30 min and 6-12 h after challenge, respectively (1). Because pretreatment of subjects with sodium cromoglycate attenuates both early and late asthmatic responses (1) and this drug has been shown in vitro to have mast cell stabilizing activity (2), it has been assumed that both early and late asthmatic responses result at least in part from release of mast cell mediators into the airways. However, there is a paucity of direct evidence for the release of mast cell mediators

This work was presented in part at the 1987 Annual Meeting of the Academy of Allergy and Immunology.

Address reprint requests to Thomas B. Casale, M.D., Department of Internal Medicine, University of Iowa Hospitals and Clinics, Iowa City, IA 52242. 1987.

Received for publication 17 April 1987 and in revised form 22 June

J. Clin. Invest.

(c) The American Society for Clinical Investigation, Inc.

$0021-9738 / 87 / 11 / 1507 / 05 \$ 2.00$

Volume 80, November 1987, 1507-1511 into the airways during antigen-induced bronchoconstriction. The purpose of the present study was, therefore, to determine if local airway challenges with antigen induces airway constriction in allergic asthmatics, and to ascertain if in vivo airway constriction is associated with measurable histamine release into the airways. We found that local antigen challenge of subsegmental airways in allergic asthmatics, but not in normal subjects, resulted in acute airway constriction and increased bronchoalveolar lavage (BAL) ${ }^{1}$ fluid histamine. The increase in BAL histamine was not present $48 \mathrm{~h}$ later.

\section{Methods}

Reagents. Histamine diphosphate, 1-methylhistamine, and Wright stains were purchased from Sigma Chemical Co., St. Louis, MO; acetone, chloroform, $\mathrm{NaOH}$, and $\mathrm{HCl}$ from Fisher Scientific Co., Pittsburgh, PA; methacholine from J. T. Baker Chemical Co., Phillipsburg, $\mathrm{NJ}: S$-[methyl ${ }^{3} \mathrm{H}$ ]adenosyl- $l$-methionine $(65-78 \mathrm{Ci} / \mathrm{mmol})$ from New England Nuclear, Boston, MA; and silica thin-layer chromatography plates (LK6D) from Whatman Inc., Clifton, NJ.

Subjects. Each participant gave informed consent to undergo BAL, aerosol antigen challenge, and local airway challenge before entering the study, which had been approved by the Human Subjects Use Committee at the University of Iowa. These studies strictly conformed to guidelines set forth by the American Thoracic Society, the American Academy of Allergy and Immunology, the American College of Chest Physicians, the National Heart, Lung and Blood Institute, and the National Institute of Allergy and Infectious Diseases (3). Subjects were adult nonsmokers between the ages of 18 and $45 \mathrm{yr}$. Each subject had a complete medical history and physical examination and was skin tested intradermally with a routine battery of antigens (Center Laboratories, Port Washington, NY). A wheal size $5 \times 5 \mathrm{~mm}$ or greater than diluent control was considered positive. Each subject also had a methacholine aerosol challenge followed by an antigen aerosol challenge that was done at least $1 \mathrm{wk}$ after methacholine challenge. Two groups were studied: allergic asthmatics and normal control subjects. The allergic asthmatics had a history of mild seasonal asthma, were skin test- and aerosol challenge-positive to the appropriate antigens, and had positive methacholine aerosol challenges. The normals had no symptoms of respiratory allergy, had negative antigen skin tests, and had negative antigen and methacholine aerosol challenges. All antigen aerosol challenges were performed at least $2 \mathrm{wk}$ before BAL. At the time of local antigen challenges and BAL, all subjects had a forced expiratory volume in $1 \mathrm{~s}\left(\mathrm{FEV}_{1}\right)>60 \%$ predicted and $\geqslant 2$ liters, and stable pulmonary functions for the preceding $3 \mathrm{wk}$.

Methacholine bronchoprovocation. Methacholine aerosol challenge was performed according to the procedure outlined by the American Academy of Allergy Committee on Standardization of Bronchoprovo-

1. Abbreviations used in this paper: BAL, bronchoalveolar lavage; $\mathrm{FEV}_{1}$, forced expiratory volume in $1 \mathrm{~s} ; \mathrm{PD}_{20} \mathrm{FEV}_{1}$, provocative methacholine cummulative dose (breath units) producing a $20 \%$ drop in $\mathrm{FEV}_{1}$; PNU, protein nitrogen unit. 
cation (4). A Johns Hopkins dosimeter was used to deliver concentrations of methacholine from 0.075 to $25 \mathrm{mg} / \mathrm{ml}$. Subjects were given five breaths of each concentration of methacholine by taking slow deep breaths from end tidal volume to total lung capacity, without breath holding. A 20\% drop in $\mathrm{FEV}_{1}$ (below diluent baseline) that persisted for at least $5 \mathrm{~min}$ was considered a positive response. The provocative dose producing a $20 \%$ decrease in $\mathrm{FEV}_{1}\left(\mathrm{PD}_{20} \mathrm{FEV}_{1}\right)$ was calculated and expressed as breath units ( 1 breath unit $=1$ inhalation of $1 \mathrm{mg} / \mathrm{ml}$ methacholine).

Antigen aerosol challenge. Antigen aerosol challenges were performed as previously described (5), and in a manner similar to the methacholine challenges. We delivered increasing fivefold concentrations of antigen from 1 protein nitrogen unit (PNU) to 10,000 PNU/ $\mathrm{ml}$. A $20 \%$ or greater drop in $\mathrm{FEV}_{1}$ that persisted at least 5 min was considered positive. Allergic asthmatics were challenged out of the appropriate season and off medications with an antigen to which they were clinically sensitive (Alternaria, ragweed, or cat extract). Normals were challenged with Alternaria (maximum dose, 10,000 PNU/ml $\times 5$ breaths).

$B A L$. All allergic asthmatics were clinically asymptomatic, out of the appropriate season, and off medications for at least $48 \mathrm{~h}$ before BAL and local antigen challenge. Fiberoptic bronchoscopy and BAL were performed as previously described $(5,6)$. Briefly, each subject was premedicated with intramuscular atropine sulfate $(0.6 \mathrm{mg})$ and morphine sulfate $(8 \mathrm{mg})$, and then received aerosolized $4 \%$ xylocaine and two puffs of metaproterenol. All subjects were intubated with a softcuff endotracheal tube. Bronchoscopy was accomplished with a flexible fiberoptic bronchoscope (BR-4B/2, Olympus Corp., New Hyde Park, NY) that was wedged into a subsegmental bronchus before BAL and local antigen challenge.

Local antigen challenge. Local antigen challenges were done as described previously (5). Antigen was diluted in warmed $\left(37^{\circ} \mathrm{C}\right)$ sterile saline and then injected through the bronchoscope in 5-ml aliquots of increasing concentration every $3 \mathrm{~min}$. In the allergic asthmatics, the initial antigen (Alternaria, ragweed, or cat extract) dose was 10-fold less than that which produced the end-point skin titration $(4 \times 4 \mathrm{~mm}$ wheal), and was based upon the PNU content of the extract. Most asthmatics and all normals received Alternaria extract prepared from the same lyophilized stock as used for aerosol challenge. Normal subjects were challenged with an initial dose of $20 \mathrm{PNU}(4 \mathrm{PNU} / \mathrm{ml})$ Alternaria and received a final amount of 100 PNU. After each dose of antigen, the airway was observed visually for $3 \mathrm{~min}$. The challenge was stopped when the airway became blanched, edematous, and constricted ( $\sim 30 \%$ or greater narrowing of airway diameter), or 100 PNU of antigen were instilled. Immediately thereafter, BAL was performed by the injection and suction of five $20-\mathrm{ml}$ aliquots of $37^{\circ} \mathrm{C}$ normal saline into the challenged subsegment. A nonchallenged control subsegment on the opposite side of the lung was challenged with diluent and lavaged in an identical manner. The same antigen-challenged and control subsegments were relavaged $48 \mathrm{~h}$ later.

Cold saline challenges. As a nonspecific challenge and an additional control for the local antigen challenges, local cold saline challenges were performed on four allergic asthmatics and seven normal subjects. The challenges consisted of the injection and suction of five 20 -ml aliquots of cold, $22^{\circ} \mathrm{C}$ normal saline into a subsegmental bronchus. A control subsegment on the opposite side of the lung was lavaged in an identical manner using warm, $37^{\circ} \mathrm{C}$ normal saline. Neither group demonstrated airway constriction in response to the cold or warm saline lavages.

$B A L$ fluid. BAL fluid was recovered in sterile traps. The lavage fluid was subsequently filtered through two layers of sterile gauze, and the cells were pelleted at $250 \mathrm{~g}$ for $5 \mathrm{~min}$. The lavage supernatants were then aliquoted and stored at $-70^{\circ} \mathrm{C}$ until assayed for histamine.

$B A L$ cell counts. Cell counts were done as described previously (5, 6). Briefly, the cells were washed, resuspended in buffer, and analyzed for total cell number using a cell counter (Coulter Electronics Inc., Hialeah, FL). Differential cell counts (expressed as percent of total cells) were obtained by counting 200 cells on a cytocentrifuged preparation stained with Wright-Giemsa.
Histamine assay. Histamine was assayed using a single isotope enzymatic assay (6) sensitive to $50 \mathrm{pg}$ histamine/ml. The assay involved extracting [ $\left.{ }^{3} \mathrm{H}\right]-1$-methylhistamine generated by the enzyme $N$-methyltransferase acting on histamine in the presence of $S$-[methyl$\left.{ }^{3} \mathrm{H}\right]$-adenosyl- $l$-methionine into chloroform, and isolating the $\left[{ }^{3} \mathrm{H}\right]-1$ methylhistamine by thin-layer chromatography. The assay used for this study has previously been shown to be very reproducible at both high and low levels of BAL histamine (6). Unknown histamine values were calculated by comparing the mean counts per minute of triplicate samples with a calibration curve of standard samples (0-2,000 pg histamine/ml) constructed by the least squares method.

Data analysis. Data were analyzed using the SAS Computer Software System (Cary, NC) on an IBM 370 computer. All results are provided as mean \pm SEM, and each statistical method employed is described in Results.

\section{Results}

All 17 allergic asthmatics had local airway constriction in response to antigen instillation through the bronchoscope. In each asthmatic subject, the initial airway response to antigen was a brief but visible blanching of the airway mucosa. This response was followed by hyperemia, and finally mucosal edema and partial airway closure. The actual amount of airway narrowing was not strictly quantitated but visually appeared to be at least $30 \%$. Despite receiving an average of nearly 100 -fold more antigen than the asthmatics, none of the nine normal subjects had blanching, edema, or narrowing of the challenged airway. The allergic asthmatics had no signs or symptoms of systemic anaphylaxis subsequent to the local challenges. None of the asthmatics noted any sensation of bronchospasm or chest tightness as a result of local antigen challenge or lavage. Immediately after local antigen challenge and BAL, the asthmatics had a clinically insignificant decrease in $\mathrm{FEV}_{1}(<15 \%)$ that returned to baseline within $15 \mathrm{~min}$. $\mathrm{FEV}_{1}$ remained at baseline values in the normal subjects.

Fig. 1 shows the BAL histamine content of the control and antigen-challenged subsegments immediately after the local challenges. The asthmatics had a mean increase in BAL histamine content of $23 \%$, which by paired sample $t$ test was significant at a $P$ value of 0.005 . In contrast, the normal subjects did not have a significant increase in BAL histamine content in response to antigen challenge. Moreover, among the allergic asthmatics, change in BAL histamine content directly correlated with control BAL histamine content (Pearson correlation coefficient $=0.66, P=0.003$ ). That is, asthmatics who had higher control BAL histamine levels responded to local antigen challenge with more marked histamine increases.

Although the mean BAL histamine content for the allergic asthmatics increased in response to local antigen challenge, some asthmatics did not have a measurable increase. We therefore compared the asthmatics without antigen-induced increases in BAL histamine $(n=8)$ to the asthmatics with measurable increases in BAL histamine $(n=9)$ to determine if antigen-induced increases in BAL histamine were associated with any other factors. Asthmatics who did not respond to antigen challenge with an increase in BAL histamine had a mean methacholine $\mathrm{PD}_{20}$ value of $41 \pm 31$ breath units, which was much greater than the $8 \pm 2$ breath units measured for asthmatics who did respond to antigen challenge with an increase in BAL histamine. However, there were no differences in the total number of percentages of BAL fluid cells between the two asthmatic groups. Thus, in allergic asthmatics, antigen-induced increases in BAL fluid histamine are associated 


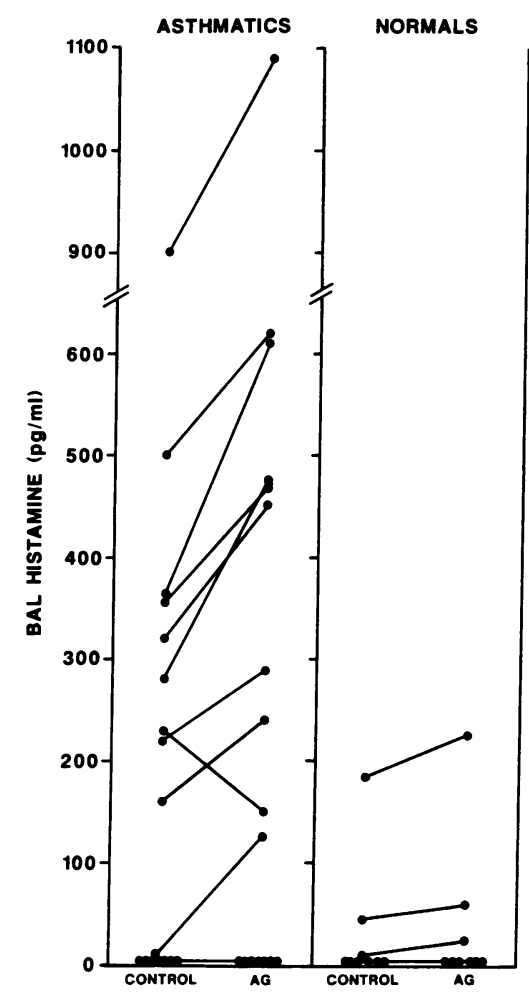

Figure 1. BAL histamine content immediately after local antigen $(A G)$ challenge. The BAL histamine content of airway subsegments challenged with antigen is compared with that measured in control airway subsegments in the opposite lung at the time of the challenge. BAL histamine content of the antigen-challenged subsegments was significantly greater $(P$ $=0.005$ by paired sample $t$ test) than BAL histamine content of the control subsegments in asthmatics but not in normals.

with increased airway responsiveness to methacholine, but not to BAL fluid cellular content.

Table I shows the BAL cell counts of the control and antigen-challenged subsegments immediately after the local challenges. Neither the asthmatics nor the normals had a significant change in BAL total cell counts or differential cell counts in response to antigen challenge. Among allergic asthmatics, the BAL histamine content was positively correlated with the number of BAL eosinophils in both the antigen-challenged (Pearson correlation coefficient $=0.57, P=0.017$ ) and control (Pearson correlation coefficient $=0.74, P=0.001$ ) subsegments. In normal subjects, however, BAL histamine content did not correlate with the number of eosinophils in either the antigen-challenged or control subsegments. Moreover, BAL histamine content did not correlate with any other cell type in either the asthmatics or normals.

$48 \mathrm{~h}$ after local antigen challenge, 13 of 17 asthmatics and 8 of 9 normals returned for a second BAL. Neither the asthmatics nor the normals had symptoms of airway constriction or changes in pulmonary functions from baseline values mea-

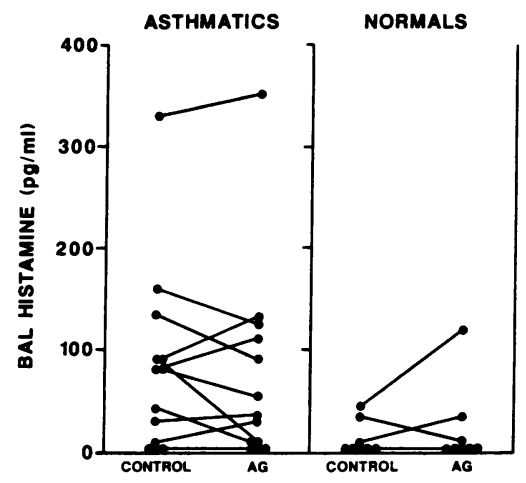

Figure 2. BAL histamine content $48 \mathrm{~h}$ after local antigen $(A G)$ challenge. BAL histamine content of airway subsegments challenged with antigen is compared with that measured in control airway subsegments in the opposite lung $48 \mathrm{~h}$ after local challenge. The BAL histamine content of the antigen-challenged subsegments was not significantly different from BAL histamine content of the control subsegments in either asthmatics or normals.

sured $48 \mathrm{~h}$ earlier. In contrast to the results obtained immediately after antigen challenge (Fig. 1), the asthmatics had no significant differences between BAL histamine levels measured in control and antigen-challenged subsegments $48 \mathrm{~h}$ after local challenge (Fig. 2). In normal subjects we found no difference between the BAL histamine levels measured in the two subsegments either immediately (Fig. 1) or $48 \mathrm{~h}$ after local challenge (Fig. 2). Thus, increases in BAL histamine levels were found only in allergic asthmatics immediately after antigen-induced airway constriction.

As an additional control for the local antigen challenges, we instilled five $20-\mathrm{ml}$ aliquots of cold, $22^{\circ} \mathrm{C}$ normal saline into the airways of four asthmatics and seven normals. Each subject also had a subsegment of the opposite lung lavaged with $37^{\circ} \mathrm{C}$ warm saline as a control. None of the subjects had airway blanching, edema, or constriction (as seen in the asthmatics after antigen challenge) in response to cold or warm saline lavages. Neither asthmatics nor normals had a significant change in BAL histamine content in response to cold saline challenge (Fig. 3). Moreover, neither group was noted to have changes in the total or differential BAL cell counts as a result of the cold saline challenges. Thus, only airway stimuli resulting in increases in BAL histamine levels were associated with airway constriction.

\section{Discussion}

Previous studies have demonstrated that antigen aerosol bronchoprovocation of allergic asthmatics is associated with an increase in circulating histamine and neutrophil chemotactic

Table I. Comparison of BAL Cell Counts in Control and Antigen-challenged Subsegments

\begin{tabular}{llllll}
\hline & Cells/ml & Macrophages & Lymphocytes & Neutrophils & Eosinophils \\
\hline & $\times 10^{3}$ & $\%$ & $\%$ & $\%$ & \\
Asthmatics $(n=17)$ & & & & & \\
$\quad$ Control & $109 \pm 11$ & $91.6 \pm 1.2$ & $5.6 \pm 0.8$ & $1.0 \pm 0.3$ & $1.8 \pm 0.9$ \\
$\quad$ Antigen & $104 \pm 9$ & $90.4 \pm 1.3$ & $6.4 \pm 0.9$ & $1.6 \pm 0.3$ & $1.6 \pm 0.7$ \\
Normals $(n=8)$ & & & & \\
Control & $117 \pm 31$ & $91.3 \pm 2.5$ & $8.3 \pm 2.4$ & $0.4 \pm 0.2$ & $0.1 \pm 0.1$ \\
Antigen & $116 \pm 23$ & $90.1 \pm 1.5$ & $9.1 \pm 1.3$ & $0.6 \pm 0.3$ & $0.1 \pm 0.1$
\end{tabular}

BAL cellular content of airway subsegments challenged with antigen is compared with that measured in control airway subsegments in the opposite lung at the time of challenge. 


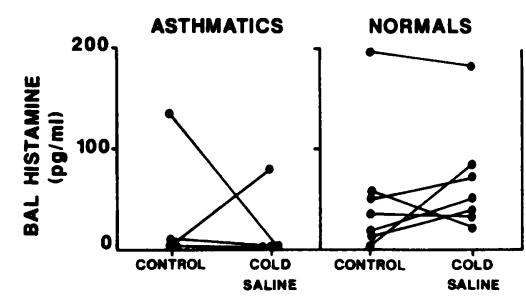

sured in control $\left(37^{\circ} \mathrm{C}\right)$ saline lavaged airway subsegments in the opposite lung at the time of challenge. BAL histamine content of the cold saline-challenged subsegments was not significantly different from the BAL histamine content of the control subsegments in either asthmatics or normals.

levels during the immediate and late asthmatic responses (7-9). Moreover, as previously discussed, sodium cromoglycate blocks both the early and late asthmatic responses (1). Although these earlier studies suggest a role for mast cells in the pathogenesis of antigen-induced bronchoconstriction, direct evidence requires the demonstration of the release of mast cell mediators into the airway lumen after airway antigen challenge. Murray and co-workers (10) preformed BAL in five asthmatics before and after local airway challenge with house dust mite. These investigators showed a significant increase in BAL fluid prostaglandin $D_{2}$ after antigen challenge in all five asthmatics. However, the airways of the asthmatic subjects were not directly visualized for airway constriction, and mediators produced exclusively by lung mast cells (e.g., histamine) were not quantitated in the BAL fluid. For this reason, we used a recently described model (5) to directly evaluate the airways of asthmatics after local antigen challenge. We observed the airways via a flexible fiberoptic bronchoscope and used BAL to evaluate changes in histamine and inflammatory cell population after local antigen challenge.

Subsequent to local antigen challenge, the airways of the asthmatics became blanched, suggesting vascular constriction. This was followed by erythema, edema, and bronchial narrowing. We could not, however, determine whether actual bronchospasm occurred. The BAL fluid obtained from these airways immediately after the onset of airway constriction contained increased levels of histamine compared with the BAL fluid from control airways in the opposite lung (Fig. 1). Despite receiving $\sim 100$-fold more antigen than the asthmatics, none of the normal subjects had local airway narrowing or significant increases in BAL fluid histamine content (Fig. 1). These data directly support the concept that exposure of sensitized airways to appropriate antigens induces the release of mast cell mediators resulting in airway constriction.

Although all 17 asthmatics had airway constriction in response to local antigen challenge, not all asthmatics had a measurable increase in BAL histamine content. The most likely explanation for not detecting an increase in BAL histamine in all 17 of the asthmatics is that the BAL histamine levels in some subjects were low at baseline, and subsequently diluted to undetectable levels by the five $20-\mathrm{ml}$ airway washes. Eight of the nine asthmatics that had baseline histamine values greater than the sensitivity of our histamine assay (i.e., $50 \mathrm{pg}$ histamine $/ \mathrm{ml}$ ) had prominent increases in BAL histamine levels measured in response to local antigen challenge. Of the eight asthmatics whose baseline BAL histamine levels were $<50 \mathrm{pg} / \mathrm{ml}$, only one had a measurable antigen-induced increase in BAL histamine. We postulate that the other seven asthmatics may also have had antigen-induced increases in BAL histamine, but the absolute histamine levels were below the detection limits of our assay. It is unclear why one of the 17 asthmatics had a decrease in BAL histamine after antigen challenge, but this may also be a reflection of the techniques used to lavage the airways.

After antigen challenge, asthmatics had a mean increase in BAL fluid histamine content of $23 \%$. If the asthmatics who did not have measurable histamine levels are excluded, the net percent histamine increase was $51 \pm 11 \%$. Moreover, the allergic asthmatics with higher control BAL histamine levels had greater antigen-induced changes in BAL histamine levels ( $r$ $=0.66, P=0.003$ ). These data suggest that asthmatics with greater baseline mast cell degranulation respond to antigen exposure with more marked mediator release.

It is possible that differences in the BAL fluid volume recovered after the five $20-\mathrm{ml}$ washes might influence the measured histamine values. Because the airways are lavaged with the same volume $(100 \mathrm{ml})$, it is reasonable to assume that the released histamine would be homogeneously diluted in that volume. Thus, the BAL fluid recovered would yield a representative aliquot reflecting the concentration of histamine in the total BAL fluid. The volume of the aliquot (i.e., the BAL fluid recovered) is probably not very important because the instilled diluent volume is the same for all samples. Moreover, by paired sample $t$ test there were no significant differences in the recovered BAL fluid volumes between the challenged and nonchallenged airways. Therefore, it is unlikely that the differences in histamine levels measured between the control and antigen-challenged subsegments in the asthmatics were affected by the BAL fluid volumes recovered.

We previously reported that allergic asthmatics had significantly greater baseline BAL histamine levels than normal subjects (6). As can be seen in Fig. 1, the baseline (control) BAL histamine levels in asthmatics were again found to be greater than the baseline BAL histamine levels measured in normal subjects. Specifically, the mean \pm SEM control BAL histamine levels were $196 \pm 60$ and $26 \pm 21 \mathrm{pg} / \mathrm{ml}$ for the allergic asthmatics and normal subjects, respectively $(P<0.05)$.

In our previous study (6) we also found that allergic asthmatics with greater baseline BAL histamine levels had more marked airway sensitivity to inhaled methacholine. In the present study, the mean methacholine $\mathrm{PD}_{20}$ value for the nine allergic asthmatics who responded to antigen challenge with a measurable increase in BAL histamine was $8 \pm 2$ breath units. However, the eight asthmatics who did not respond to antigen challenge with a measurable increase in BAL histamine had a mean methacholine $\mathrm{PD}_{20}$ value of $41 \pm 31$ breath units. Antigen-induced increases in BAL histamine levels in allergic asthmatics were not associated with changes in any other measured parameter. Significant increases in methacholine airway responsiveness have been reported to occur in asthmatics after antigen inhalation (11), and cholinergic nervous system stimulation has been shown to potentiate the response to antigen challenge in central airways through augmented mast cell mediator release (12). These data suggest that mast cell mediators released in the airways could enhance cholinergic transmission and also contribute to both bronchial hyperreactivity and the augmentation of further antigen-induced mast cell degranulation.

Immediately after local antigen challenge, neither the asthmatics nor the normals had a significant change in the BAL total or differential cell counts (Table I). Moreover, with the 
exception of eosinophils, the control (baseline) BAL cell counts were not significantly different between asthmatics and normals. In this study, as in our previous study (6), allergic asthmatics were found to have significantly greater $(P<0.05)$ BAL eosinophils $(1.8 \pm 0.9 \%)$ than normal subjects $(0.1 \pm 0.1 \%)$. However, in contrast to both our previous results (6) and those by Rankin and co-workers (13), we found that among the allergic asthmatics there was a positive correlation between number of BAL eosinophils and BAL histamine content (both in the control and antigen-challenged subsegments). The reason for this discrepancy is not clear, but may be due to subject differences between these studies. Because eosinophil products can release mediators from mast cells (14) and the mast cell produces chemotactic factors for eosinophils (15), it is not surprising to find that there is a positive correlation between the content of BAL histamine and the number of BAL eosinophils.

To determine if the immediate antigen-induced changes in BAL histamine content persisted for longer periods, we relavaged many of the asthmatics and normal subjects $48 \mathrm{~h}$ after local antigen challenge. Neither the asthmatics nor the normals had an increase in BAL histamine at this later time point. Due to a presumed increased risk to patients, we did not relavage asthmatics at the expected peak of the late asthmatic response. Thus, we are unable to comment whether there might be a second release of mediators in response to airway antigen challenge at 3-12 h, as reported after nasal antigen provocation (16).

As an additional control for the local antigen challenges, we instilled cold, $22^{\circ} \mathrm{C}$ normal saline into the airways of asthmatic and normal subjects. Neither the asthmatics nor the normals had airway constriction, increases in BAL histamine (Fig. 3), or changes in BAL total or differential cell counts in response to cold saline challenges.

In summary, local antigen challenges resulted in airway constriction and histamine release in allergic asthmatics, but not in normals. Larger antigen-induced increases in BAL histamine content were associated with higher baseline BAL histamine levels and greater airway methacholine sensitivity. Our studies provide direct evidence that when exposed to clinically significant and relevant antigens, allergic asthmatics have in vivo lung mast cell degranulation and mediator release resulting in airway contractility and contributing to airway hyperresponsiveness.

\section{Acknowledgments}

The authors wish to express their appreciation to Pam Iwamota and Marti Monick for their expert technical assistance, and to Penny L. Dean and Darla Bartels for their secretarial assistance.

This work was supported in part by an Asthma and Allergic Diseases Center grant, AI-19093 NIAID, and grant RR59, General Clinical Research Center Branch, National Institutes of Health.

\section{References}

1. Booij-Noord, H., N. G. M. Orie, and K. DeVries. 1971. Immediate and late bronchial obstructive reactions to inhalation of house dust and protective effects of disodium cromoglycate and prednisone. $J$. Allergy Clin. Immunol. 48:344-353.

2. Leung, K. B. P., K. C. Flint, J. Brostoff, B. N. Hudspith, N. M. Johnson, and F. L. Pierce. 1986. Some properties of mast cells obtained by human bronchoalveolar lavage. Agents Actions. 18:110-112.

3. Bernstein, I. L., H. A. Boushey, R. M. Cherniack, J. N. Fink, J. D. Fulmer, E. J. Goetzl, R. A. Goldstein, S. S. Hurd, S. J. Ram, L. M. Lichtenstein, H. V. Reynolds, R. M. Senior, R. A. Simon, P. Ward, and D. C. Zavala. 1985. Summary and recommendations of a workshop on the investigative use of fiberoptic bronchoscopy and bronchoalveolar lavage in asthmatic patients. Chest. 88:136-138.

4. Chai, H., R. S. Farr, L. A. Froelich, D. A. Mathison, J. A. McLean, R. R. Rosenthal, A. L. Sheffer II, S. L. Spector, and R. G. Townley. 1975. Standardization of bronchial inhalation challenge procedures. J. Allergy Clin. Immunol. 56:323-327.

5. Metzger, W. J., D. Zavala, H. B. Richerson, P. Moseley, P. Iwamota, M. Monick, K. Sjoerdsma, and G. W. Hunninghake. 1987. Local allergen challenge and bronchoalveolar lavage of allergic asthmatic lungs: description of the model and local airway inflammation. Am. Rev. Respir. Dis. 135:433-440.

6. Casale, T. B., D. Wood, H. B. Richerson, S. Trapp, W. J. Metzger, D. Zavala, and G. W. Hunninghake. 1987. Elevated bronchoalveolar lavage fluid histamine levels in allergic asthmatics are associated with methacholine bronchial hyperresponsiveness. J. Clin. Invest. 79:1197-1203.

7. Agius, R. M., P. H. Howarth, M. K. Church, C. Robinson, and S. T. Holgate. 1986. Luminal mast cells of the human respiratory tract. In Mast Cell Differentiation and Heterogeneity. A. D. Befus, J. Bienenstock, and J. A. Denburg, editors. Raven Press, New York. 277-288.

8. Durham, S. R., T. H. Lee, O. Cromwell, R. J. Shaw, T. G. Merrett, J. Merrett, P. Cooper, and A. B. Kay. 1984. Immunologic studies in allergen-induced late-phase asthmatic reactions. J. Allergy Clin. Immunol. 74:49-60.

9. Metzger, W. J., H. B. Richerson, and S. I. Wasserman. 1986. Generation and partial characterization of eosinophil chemotactic activity and neutrophil chemotactic activity during early and late-phase asthmatic response. J. Allergy Clin. Immunol. 78:282-290.

10. Murray, J. J., A. B. Tonnel, A. R. Brash, L. J. Roberts, P. Gosset, R. Workman, A. Capron, and J. A. Oates. 1986. Release of prostaglandin $\mathrm{D}_{2}$ into human airways during acute antigen challenge. N. Engl. J. Med. 315:800-804.

11. Bar-Sela, S., D. P. Schlueter, S. R. Kitt, A. J. Sosman, and J. N. Fink. 1985. Antigen-induced enhancement of bronchial reactivity. Chest. 88:114-116.

12. Leff, A. R., N. P. Stimler, N. M. Munoz, T. Shioya, J. Tallet, and C. Dame. 1986. Augmentation of respiratory mast cell secretion of histamine caused by vagus nerve stimulation during antigen challenge. J. Immunol. 136:1066-1073.

13. Rankin, J. A., M. Kaliner, and H. Y. Reynolds. 1987. Histamine levels in bronchoalveolar lavage from patients with asthma, sarcoidosis, and idiopathic pulmonary fibrosis. J. Allergy Clin. Immunol. 79:371-377.

14. O’Donnell, M. C., S. J. Ackerman, G. J. Gleich, and L. L. Thomas. 1983. Activation of basophil and mast cell histamine release by eosinophil major basic protein. J. Exp. Med. 157:1981-1991.

15. Goetzl, E. J., and K. F. Austen. 1975. Purification and synthesis of eosinophilotactic tetrapeptides of human lung tissue: identification as eosinophil chemotactic factor of anaphylaxis. Proc. Natl. Acad. Sci. USA. 72:4123-4127.

16. Togias, A., R. M. Naclerio, D. Proud, C. Baumgarten, S. Peters, P. S. Creticos, J. Warner, A. Kagey-Sobotka, N. F. Adkinson, P. S. Norman, and L. M. Lichtenstein. 1985. Mediator release during nasal provocation: a model to investigate the pathophysiology of rhinitis. Am. J. Med. 79(Suppl. 6A):26-33. 